\title{
Controlled ovarian stimulation and oocyte retrieval under continuous administration of Dienogest in a patient with catamenial pneumothorax
}

\author{
Tsuyoshi Takiuchi*, Mika Handa, Tatsuya Miyake and Tadashi Kimura \\ Department of Obstetrics and Gynecology Graduate School of Medicine, Osaka University, Japan
}

\begin{abstract}
Background: Catamenial pneumothorax $(\mathrm{CP})$ is a rare disease that accompanies recurrent pneumothorax in conjunction with menstrual cycles and is presumed to be caused by endometriosis. Dienogest, a selective progesterone receptor agonist, has been used as the long-time treatment for endometriosis. However, the effect of dienogest on oocyte growth during controlled ovarian stimulation (COS) remains unclear.
\end{abstract}

Case: We present the case of a 40-year-old nulliparous, infertile woman with CP who underwent COS and oocyte retrieval under continuous administration of dienogest.

Conclusion: Our experience suggests that continuous administration of dienogest during COS is effective for the prevention of CP recurrence without impairing the outcome of COS.

\begin{abstract}
Abbreviations: CP: Catamenial pneumothorax; COS: Controlled ovarian stimulation
\end{abstract}

\section{Introduction}

Catamenial pneumothorax $(\mathrm{CP})$ is a rare disease that accompanies recurrent pneumothorax in conjunction with menstrual cycles and is presumed to be caused by endometriosis [1]. The standard treatment for $\mathrm{CP}$ is multidisciplinary, consisting of surgical treatment and hormone therapy. Dienogest, a selective progesterone receptor agonist, has been used as the long-time treatment for endometriosis [2]. However, the effect of dienogest on oocyte growth during controlled ovarian stimulation (COS) remains unclear, and few cases about COS under continuous administration of dienogest have been reported. Here, we report a case of COS and oocyte retrieval under continuous administration of dienogest in an infertile woman with CP.

\section{Case report}

A 40-year-old nulliparous, infertile woman with an Anti-Müllerian Hormone (AMH) value of $1.44 \mathrm{ng} / \mathrm{mL}$ was referred to our hospital for infertility treatment. Six years ago, she experienced a right-sided pneumothorax during her menstrual cycle and five episodes of recurrent right-sided pneumothorax during menstrual cycles for subsequent seven months, followed by thoracoscopic surgery. She was strongly suspected to have $\mathrm{CP}$, according to her clinical history and operative findings, including many holes in the diaphragm, although the histopathology did not clearly reveal endometrial tissue in the resected specimen. No recurrence of $\mathrm{CP}$ had been observed since the administration of dienogest $2 \mathrm{mg}$ daily after the surgery.

Based on a consultation with the Department of Thoracic Surgery in our hospital, continuation of dienogest during the infertility treatment was recommended, weighing the increased risk of recurrence of CP due to the withdrawal of dienogest. Therefore, we chose randomstart ovarian hyperstimulation under continuous administration of dienogest to retrieve twelve oocytes, seven of which were metaphase II oocytes (Figures 1 and 2). Finally, three day 3 embryos and one blastocyst were cryopreserved. She did not suffer from CP during or after COS.

\section{Discussion}

$\mathrm{CP}$ is a rare disease, but it may be underdiagnosed. A report indicated that it is more frequent than expected because it was diagnosed in almost $25 \%$ of women who had undergone surgical treatment with spontaneous pneumothorax [1]. The clinical presentations and possible associated predictive factors have not been described in detail. Pelvic endometriosis was found in $51 \%$ of women with CP, and infertility and uterine procedures are significantly associated with $\mathrm{CP}$, although the relationship between endometriosis and infertility has already been recognized [3]. Lalani et al. reported that dienogest may be an effective hormonal treatment option and alternative to long-term $\mathrm{GnRH}$ agonist therapy for women with recurrent CP [4].

Dienogest, a selective progesterone receptor agonist, has been widely used for the treatment of endometriosis in women of reproductive age who may suffer from infertility [2]. Therefore, it is not rare to treat infertile patients with dienogest, but there has been some

${ }^{\star}$ Correspondence to: Tsuyoshi Takiuchi, Department of Obstetrics and Gynecology Graduate School of Medicine, Osaka University, Japan, Tel: 81-66879-3356; Fax: 81-6-6879-3359; E-mail: takkitakktakki@gyne.med.osaka-u.ac.jp

Key words: dienogest, controlled ovarian stimulation, catamenial pheumothorax Received: December 05, 2019; Accepted: December 12, 2019; Published: December 16, 2019 


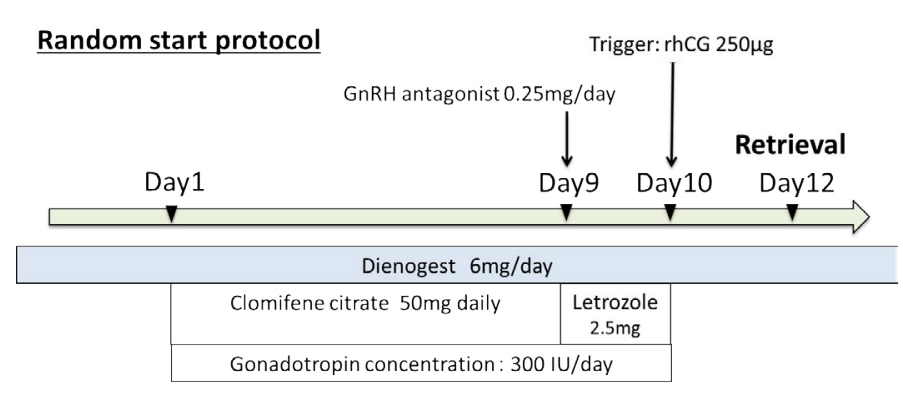

Figure 1. Treatment scheme for the random start protocol

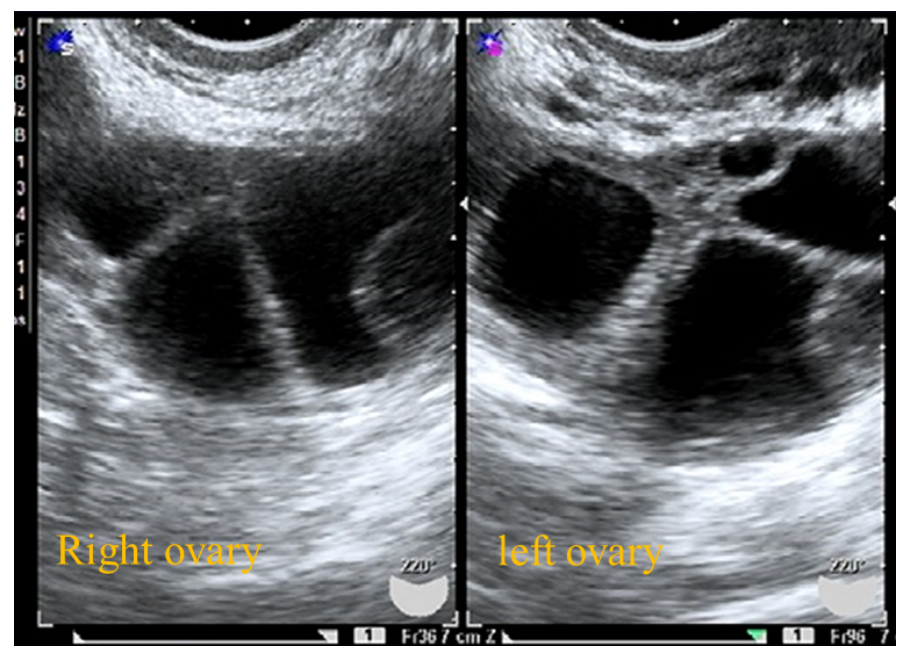

Figure 2. Transvaginal ultrasound showing both ovaries on day 10

concern that the local effects of dienogest may have a direct impact on follicular maturation or that the systemic levels may be high enough to affect ovarian stimulation. The effect of dienogest on COS has not been reported yet, although other progestins have been studied. A study to evaluate differences in ovarian stimulation outcomes in women using a levonorgestrel-releasing intrauterine device (LNG-IUD) demonstrated that LNG-IUDs do not affect cycle performance, including total oocyte or mature oocyte yields, in women undergoing ovarian stimulation cycles [5]. Kuang, et al. showed that in the medroxyprogesterone acetate (MPA) group (progestin primed ovarian stimulation), the number of oocytes retrieved, and mature oocytes was similar to that in the short protocol group for women undergoing COS [6]. These data about other progestins do not indicate adverse effects related to dienogest with respect to reproductive outcomes. However, dienogest should not be continued unless its necessity during COS has been assessed by a multidisciplinary team. Although there are no factors to predict the recurrence of $\mathrm{CP}$, the increased risk of recurrence of $\mathrm{CP}$ owing to the withdrawal of dienogest should be considered. Our results showed that the number of oocytes retrieved was sufficient despite the age and $\mathrm{AMH}$ value, although the proportion of immature oocytes was slightly higher than usual. Further investigation is warranted.

\section{Conclusion}

Our experience suggests that continuous administration of dienogest during COS was effective for the prevention of $\mathrm{CP}$ recurrence without impairing the outcomes of COS.

\section{Financial support}

None.

\section{References}

1. Alifano M, Roth T, Broët SC, Schussler O, Magdeleinat P, et al. (2003) Catamenial pneumothorax: a prospective study. Chest 124: 1004-1008.

2. Schindler AE (2011) Dienogest in long-term treatment of endometriosis. Int J Womens Health 3: 175-184.

3. Rousset-Jablonski C, Alifano M, Plu-Bureau G, Camilleri-Broet S, Rousset P, et al (2011) Catamenial pneumothorax and endometriosis-related pneumothorax: clinical features and risk factors. Hum Reprod 26: 2322-2329.

4. Lalani S, Black A, Hodge MC, Tulandi T, Chen I (2017) Dienogest Therapy as Treatment for Catamenial Pneumothorax: Case Report and Review of Hormonal Options. J Obstet Gynaecol Can 39: 764-768.

5. Adeleye AJ, Aghajanova L, Kao CN, Cedars MI, Sauer MV (2018) Impact of the levonorgestrel-releasing intrauterine device on controlled ovarian stimulation outcomes. Fertil Steril 110: 83-88.

6. Kuang Y, Chen Q, Fu Y, Wang Y, Hong Q, et al. (2015) Medroxyprogesterone acetate is an effective oral alternative for preventing premature luteinizing hormone surges in women undergoing controlled ovarian hyperstimulation for in vitro fertilization. Fertil Steril 104: 62-70.

Copyright: (C2019 Takiuchi T. This is an open-access article distributed under the terms of the Creative Commons Attribution License, which permits unrestricted use, distribution, and reproduction in any medium, provided the original author and source are credited. 